\title{
American English-Speakers' Perception of Non-Native Phonotactic Constraints: The Influence of Training in Phonology
}

\author{
Bailey Pearson, Kimberly Frazier \\ University of Arkansas, Arkansas, United States
}

\begin{abstract}
The purpose of the present study was to examine the differences between perceptions of non-native phonotactic rules and constraints by monolingual English-speaking undergraduate students in a program of communication disorders who had taken and passed a course in the study of phonology and by undergraduate students in communication disorders who had not yet taken a course in phonology. Participants listened to audio recordings of words from Hindi, Hmong, Kurdish, Russian, and Swedish recorded by speakers fluent in those languages. Each of the words contained at least one phonotactic constraint that is not permitted in American English phonology. Participants were instructed to write exactly what they heard after each word in the recordings, and their perceptions of the illegal constraints were scored as correct or incorrect. No significant difference was found between the students who had taken a phonology course and the students who had not. The most common misperception made was the omission of one phoneme when two were illegally combined. The results of this study, though not consistent with anticipated results, have many implications for issues concerning the linguistic diversity of the United States, among other issues related to language.
\end{abstract}

Keywords: phonology, phonotactic constraints, foreign languages, speech-language pathology

\section{Introduction}

Phonology is defined as the study of the speech sound system of a language, which includes the rules for combining and using phonemes, or speech sounds. In accordance with this definition, every established language around the world has its own unique set of phonemes and rules of phonology (or phonotactic constraints). As a result of the uniqueness of the speech sounds and phonotactic constraints that constitute different languages, it can often be difficult for non-native listeners of a language to accurately decipher the sounds of a word produced by a speaker of the given language. Researchers have studied this phenomenon in great depth among many different languages, resulting in discoveries, such as possible explanations for it and methods through which it can be overcome (D. E. Callan, Jones, A. M. Callan, \& Akahane-Yamada, 2004; Lentz \& Kager, 2015; Onishi, Chambers, \& Fisher, 2002; Polka, 1992). However, up to this point in time, no research has been conducted to assess whether or not individuals who have an extensive amount of knowledge in the study of phonology will be as susceptible to this phenomenon as those without such knowledge. Given previous research that has found the superior phonological awareness skills of speech-language pathologists in

Bailey Pearson, B.S.E, Department of Rehabilitation, Human Resources, and Communicaiton Disorders, University of Arkansas, Arkansas, USA.

Kimberly Frazier, Ph.D., Associate Professor, Department of Rehabilitation, Human Resources, and Communication Disorders, University of Arkansas, Arkansas, USA. 
comparison to other professionals (Spencer, Schuele, \& Guillot, 2008; Messier \& Jackson, 2014), this is a question worthy of investigation.

Regarding underlying causes for non-native listeners' phoneme misperceptions, the research has shown that phonological categorical knowledge may facilitate perceptions of non-native speech sounds. For example, in a study comparing English and Farsi listeners of the Salish language, Polka (1992) found that phonetic familiarity as well as acoustic characteristics may be contributing factors to these perceptions. Neither English nor Farsi possess phonetic categories similar to Salish, so the fact that English-speaking participants and Farsi-speaking participants had an equal amount of difficulty perceiving the Salish speech sounds presented in the task suggests that phonetic familiarity may have an influence on the misperceptions non-native listeners make (Polka, 1992). Similarly, Lentz and Kager (2015) conducted a study that sought to determine the influence that categorical phonotactic knowledge (i.e., knowledge of phonotactic constraints) of one's first language has on second language input. This study compared first language (L1) Spanish speakers, Japanese speakers, and other language speakers who spoke Dutch as a second language (L2) to L1 Dutch speakers concerning their accuracy and reaction time in performing a lexical decision task (a task in which participants discern words from non-words) with Dutch words (Lentz \& Kager, 2015). Lentz and Kager (2015) proposed that if L1 phonotactic knowledge governs L2 perceptions, then an individual's ability to acquire L2 phonotactic knowledge will be hindered. Participants' results on the task were consistent with what Lentz and Kager (2015) proposed as the "Leaky Filter" scenario, in which the L1 phonotactic filter (i.e., the phonotactic knowledge of the L1 that restricts illegal phonological contrasts) exists, but it is not so powerful as to prevent acquisition of L2 phonotactic knowledge completely.

A study by Onishi et al. (2002) discovered a similar phenomenon. In this study, researchers considered the ability of native English speakers to acquire knowledge of phonotactic constraints not present in the English language after having briefly studied them (Onishi et al., 2002). In three experiments, Onishi et al. (2002) assessed the influences of: (a) new consonant position restrictions; (b) new consonant positions dependent on adjacent vowels; and (c) consonant positions dependent on the speaker's voice on participants' ability to learn novel phonotactic constraints. In each experiment, participants were assigned to listen to various lists of CVC (consonant-vowel-consonant) syllables and were then tested on their perceptions of three different stimuli: (a) items that were on their study lists; (b) items that were phonotactically illegal: and (c) items that were phonotactically legal (Onishi et al., 2002). Overall, participants' results in the three experiments displayed that new phonotactic constraints from unfamiliar languages can indeed be learned through brief auditory exposure (Onishi et al., 2002).

The implications of the research findings discussed thus far are such that an individual's background of their first language establishes a pathway for their understanding of other languages and dictates the perceptions they make when listening to other languages. However, it does so without completely preventing acquisition of non-native phonological knowledge and perceptual skills. Against this background, it is reasonable to inquire of the abilities of individuals who have been trained in phonology to more accurately perceive native phonemes in non-native positions and combinations at the first exposure. Furthermore, the combination of these findings with those that provide evidence for speech-language pathologists' superior phonological knowledge and skills make for an even more compelling research question.

In a study by Spencer et al. (2008), speech-language pathologists (SLPs) were found to display phonemic awareness skills (i.e., skills in hearing, identifying, and manipulating phonemes) that were significantly more 
advanced than those of other educators, who had not been trained extensively in the study of phonology. Using a measure that assessed phoneme segmentation, phoneme identification, and phoneme isolation, Spencer et al. (2008) found that SLPs performed significantly better than kindergarten teachers, first-grade teachers, reading teachers, and special education teachers on phonemic awareness application tasks. Additionally, Messier and Jackson (2014) found that, when compared to teachers of the deaf (TODs), speech-language pathologists who work with children who are deaf or hard of hearing performed significantly better on average on the Phonological and Phonemic Awareness Measure (PPAM), though the two groups reported similar levels of confidence in their phonological awareness skills. While both TODs and SLPs are required to have advanced training in order to work with children who are deaf or hard of hearing, according to the American Speech-Language-Hearing Association (ASHA, 2004), the two groups differ in some areas of their training, phonology likely being one of those areas (as cited in Messier \& Jackson, 2014). Based on the findings of these studies, it is reasonable to conclude that a study comparing phonological skills between communication disorders students who have passed a course in phonology and students who have not would generate similar results.

The primary purpose of this study was to examine the influence that an understanding of the study of phonology has on an individual's perceptions of non-native phonotactic constraints. Considering the ever-changing linguistic demographics of the United States, it is important to understand what drives our perceptions of other languages and how we can improve as a society in terms of embracing linguistic diversity. Two secondary aims of the study included: (a) determining whether some of the languages used in the study contain phonotactic constraints that are easier for native English-speakers to perceive than other languages; and (b) identifying the most common misperceptions of foreign phonotactic constraints that native English-speakers make. An a priori hypothesis was such that individuals who had a foundational knowledge of the study of phonology would more accurately perceive native speech sounds in non-native positions and combinations than individuals who did not.

\section{Method}

\section{Participants}

Participants in this study included 35 undergraduate students majoring in Communication Disorders at the University of Arkansas. According to the University of Arkansas College of Education and Health Professions website, students in the program of Communication Disorders are required to take a course in the study of normal phonology during their first semester in the program (see http://cdis.uark.edu). In this course, students learn the characteristics of normal speech sounds present in the English language, the articulatory positions and movements involved in the production of speech, and how to transcribe speech using the International Phonetic Alphabet (IPA), a method of transcription used by professionals in the field of communication disorders (see http://cdis.uark.edu). Consequently, students who pass this course (in terms of the program criteria) are considered to have a substantial amount of knowledge in phonology. Of the 35 participants, 13 were students who had not yet taken this phonology course (Group A) and 22 were students who had taken and passed the course (Group B). All participants were monolingual native English-speaking, with no reported diagnoses of hearing difficulties. Recruitment was executed via word of mouth and email. The first round of participants was compensated with a meal for lunch, and the second round was provided with donuts for breakfast. All participants were required to give written informed consent as approved by the University of Arkansas Institutional Review Board. 


\section{Materials}

Five individuals (one faculty member in the Department of Rehabilitation, Human Resources, and Communication Disorders, one staff member in the Engineering Research Center, one staff member in the Department of Research \& Service Units, and two students in the graduate program of Speech-Language Pathology at the University of Arkansas), each fluent in one of this study's target languages (Hindi, Hmong, Kurdish, Russian, and Swedish), assisted in this study. The specific languages were chosen as target languages for the study through the focus of having a variety of world languages as well as the result of the researchers having convenient access to fluent speakers of those languages. Each individual, with the assistance of the study's primary researcher, compiled a list of words in their respective language that contained at least one phonotactic constraint that is permitted in their language but not permitted in the English language (e.g., / $3 /$ in the initial position of a word, / $\mathrm{f} /$ in the initial position of a word, etc.). No foreign phonemes were included within the foreign constraints of the words used; each word possessed phonemes that were native to both English and the respective foreign language. They were then recorded via an audio recorder speaking each of the words with about a five-second pause between each word. These five audio recordings, with a total of 84 test items, were used to assess participants' perceptions of non-native phonotactic constraints. Validity and reliability of the materials was ensured by using native or experienced speakers of the five languages.

\section{Procedures}

Data was initially collected from 32 participants. The participants were seated in a classroom with noise-absorbing acoustic panels on the walls and were given an answer sheet on which to transcribe their responses. They were instructed to "write down exactly what [they heard] after each word". Group A (individuals who had not yet taken a phonology course) was instructed to use English graphemes to transcribe their perceptions of the words, while Group B (individuals who had taken and passed phonology) was instructed to use the International Phonetic Alphabet. To ensure correct interpretation of Group A's responses, a brief training session was conducted to clarify which graphemes they should use to represent which sounds for those sounds in English that could be represented by multiple graphemes (e.g., "k" was to be used to represent the $/ \mathrm{k}$ / sound, rather than "c" or "ck"). All five recordings were played for them in alphabetical order of the language, and they were notified between the languages when a new language was about to be presented. In an attempt to recruit more participants for Group A, another round of experimentation was held three weeks later, in which three more participants completed the study. This group was given the exact instructions as the first group, and the training session was also conducted prior to experimentation.

\section{Results}

\section{Objective 1: Effect of Training in Phonology}

The participants' responses for each illegal constraint was scored as either correct or incorrect, and each participant was given an overall percentage score of their number of correct responses out of the total number of test items. To address the primary question of the study, scores were compared between the two groups using an independent group's $t$-test. Results indicated that there was no significant difference between Group A ( $M=$ $18.7, S D=9.68)$ and Group B $(M=17.6, S D=7.01)$ in terms of their abilities to correctly perceive non-native phonotactic constraints overall, $\mathrm{t}(33)=0.36, p>0.05$. Scores were again compared using the independent group's $t$-tests to determine if there were differences for each of the individual languages between the two 
groups, and no significant differences appeared for any of the languages. Group mean scores for each of the languages for each group and the independent group's $t$-test results appear in Table 1.

Table 1

Participant Mean Scores for the Five Languages

\begin{tabular}{llll}
\hline & Group A mean & Group B mean & $T$-test $p$-value \\
\hline Hindi & 18 & 18.2 & 0.9707 \\
Hmong & 8.7 & 8.1 & 0.8481 \\
Kurdish & 20.4 & 19.8 & 0.8832 \\
Russian & 22.3 & 24.5 & 0.6065 \\
Swedish & 28.2 & 19 & 0.0549 \\
\hline
\end{tabular}

Note. Group mean scores are the average percent of the number of correct responses out of the total number of responses for the participants in each group.

\section{Objective 2: Effect of Language}

Two analyses of variance were conducted to determine, within each group, if the participants found one language's constraints significantly easier to perceive than the other languages'. For Group A, the language did not influence the participants' abilities to correctly perceive non-native phonotactic constraints at the $p<0.05$ level, $F(4,59)=0.3142, p=0.13$. Similarly, the language did not have a significant effect on Group B's abilities to correctly perceive non-native phonotactic constraints at the $p<0.05$ level, $\mathrm{F}(4,105)=0.2807, p=$ 0.11. Both groups yielded the lowest average scores for the Hmong language. However, there was a discrepancy between the two groups in terms of the language for which they had the most correct perceptions, as Group A performed the best for the Swedish language, while Group B performed the best for Russian.

\section{Objective 3: Common Misperceptions}

The most common misperception made by participants was the omission of a phoneme when two were illegally combined (e.g., reducing /dd/ to /d/ and /sv/ to /s/). The second most common misperception was the addition of a vowel (i.e., epenthesis) between two consonants that were illegally combined in a specific position (e.g., $/ \mathrm{dd} /$ in the initial position to $/ \mathrm{d} \wedge \mathrm{d} /$ and $/ \mathrm{bn} /$ in the initial position to $/ \mathrm{bin} /$ ). Other common misperceptions included: (a) perceiving the cognate of a target phoneme (i.e., the phoneme that possesses all the same phonetic characteristics as the target phoneme, but differs only in voicing) rather than the target phoneme (e.g., /p/ instead of $/ \mathrm{b} /, / \mathrm{s} /$, instead of $/ \mathrm{z} /$ ) in order to make a legal constraint; and (b) perceiving a phoneme that possessed the same manner of articulation as the target phoneme (e.g., $/ \mathrm{n} /$ instead of $/ \mathrm{m} /, / \mathrm{t} /$, instead of $/ \mathrm{k} /$ ) in order to make a legal constraint. Raw data for the number of occurrences of each of these types of misperceptions for groups A and B appear in Table 2.

Table 2

Total Occurrences of Common Phonotactic Constraint Misperceptions

\begin{tabular}{lllll}
\hline & Omission & Epenthesis & Cognate & Same manner \\
\hline Group A & 235 & 60 & 28 & 32 \\
Group B & 428 & 136 & 40 & 30 \\
Total & 663 & 196 & 68 & 62 \\
\hline
\end{tabular}

Notes. For the purposes of this study, omission was defined as the removal of one consonant when two were illegally combined. Epenthesis was defined as the insertion of a vowel between two consonants that were illegally combined. Misperceptions with the label cognate were assigned when the participants perceived the cognate of a target phoneme rather than the target to make a legal phonotactic constraint. Misperceptions with the label phoneme with same manner were assigned when the participants perceived a phoneme with the same manner of articulation as the target phoneme (excluding cognates) rather than the target to make a legal constraint. 


\section{Discussion}

\section{Objective 1: Effect of Training in Phonology}

According to the results from the independent samples $t$-tests, knowledge of phonology does not significantly improve native American English-speakers' overall abilities to correctly perceive non-native phonotactic constraints. Group B's mean percentage score was not significantly higher than Group A's for all the languages combined or between each of the individual languages. In fact, though not statistically significant, the data almost yielded a reverse effect in that Group A's overall mean percentage score was slightly higher than that of Group B. Additionally, the only languages for which Group B yielded a higher mean percentage score than did Group A were Hindi and Russian. The researchers hypothesize that this effect may have occurred as a result of a heavier cognitive load being placed on the participants in Group B, as they were asked to transcribe using IPA rather than using English graphemes. The participants in Group A may have had an advantage in that they were able to use a more familiar method of transcription, which could have reduced the possibility of simple errors in transcription-an issue to which Group B may have been more susceptible. However, the results may also simply suggest that knowledge and perceptibility of phonotactic constraints is innate and firmly established by knowledge of a person's first language. Consequently, awareness of foreign phonotactic constraints at the first exposure will not be significantly improved by a person's knowledge of American English phonology. It should also be noted that the participants who had taken the phonology course studied the phonology of American English only. While this may have improved their phonological awareness skills for English, such skills may not have been able to transfer across languages.

\section{Objective 2: Effect of Language}

According to the results from the two analyses of variance, type of language had no significant effect on either group's ability to correctly perceive foreign phonotactic constraints. Both groups yielded the lowest scores for Hmong. However, Group A performed the best for Swedish, while Group B performed the best for Russian. It is possible that this discrepancy is rooted in the no phonological knowledge vs. phonological knowledge variable; future researchers would do well to look into this possibility. Additionally, it is possible that degree of accent of the speakers introduced a confounding variable into the study, as some speakers possessed a greater native speaking accent than others. For example, the Hmong speaker had a relatively heavy native accent, as she was a native speaker of the Hmong language. However, the Russian speaker, though fluent in Russian, was a native American English speaker and, therefore, did not have a native Russian accent. Perhaps this variable inadvertently affected the participants' perceptual abilities in addition to the variable of interest, the foreign phonotactic constraints.

\section{Objective 3: Common Misperceptions}

It is believed that the most significant implications of this research are found in the results of this final objective-identifying the most common misperceptions that the participants made while listening to the foreign words. First, having a more robust understanding of these misperceptions has possibly great ramifications for issues that arise with the collection of diverse linguistic identities that are present across the United States. For example, individuals who are teaching English to English-language learners (ELLs) could benefit from an awareness of the fact that phonotactic constraints differ across languages and that those discrepancies are often difficult for non-native listeners to perceive. With this knowledge as a foundation, individuals teaching English to ELLs may be able to more accurately identify and direct focused attention to 
any phonotactic constraints unique to the English language that are particularly difficult for the ELL to perceive. This is an important aspect of language learning to consider because, while the language-learning individual may be able to attribute meaning to the new words they are learning, they will not have a completely proficient understanding until they have acquired awareness of the phonological compositions of those words. Such abilities would result in the individual's more thorough understanding of the language's phonology overall, which would serve as a firm foundation on which further linguistic knowledge could be built.

Taking into account the languages for which the groups performed the least and most accurately in this study may serve as a guide through which expectations for success in perceiving and learning new phonotactic constraints could be based. Individuals may expect, for example, ELLs who primarily speak a European language (e.g., Russian or Swedish) to perceive and subsequently learn English phonotactic constraints more easily than ELLs who primarily speak an Asian language (e.g., Hmong). However, this prediction assumes that the results of this study would be replicated in the case of non-English speakers perceiving English phonotactic constraints-the converse of what was studied in this research. Further research would be necessary to support this assumption.

\section{Limitations}

There are a few limitations with the present study to be addressed. First, while the goal was to recruit at least 20 participants for each group, only 13 individuals for Group A responded to the call for participants. However, over 20 individuals for Group B took part in the study, which resulted in a difference of nine participants between the two groups. It is possible that the relatively small sample size for Group A resulted in average responses that were less representative of their population than that of Group B. Another limitation of the study is the differences in method of transcription between the two groups of participants. As previously mentioned, it is possible that requiring participants in Group B to use the International Phonetic Alphabet (IPA) placed a heavier cognitive load on them than was placed on the participants in Group A. Subsequent studies may consider requiring the participants to verbally repeat their perceptions aloud to be transcribed by the experimenters rather than transcribing them themselves. This design may also help control for an issue that was faced by the researchers while analyzing the responses, which was an occasional handwriting illegibility problem. While this issue was not encountered but a few times, it often occurred in instances where the illegible symbol was determinant of whether the perception would be scored as correct or incorrect. Controlling for this issue would yield more objective results and aid researchers in the process of analysis. Another limitation of the study was the confounding variable of degree of spoken accent for each speaker. As mentioned in the discussion, the five speakers had differing degrees of accent, some being native speakers of the foreign languages and some not. It is unclear whether or not this variable had a significant influence on the participants' perceptions, but recruiting, for example, all native speakers of the five languages to assist in the study would have increased validity and reliability of the materials, and consequently, made for a more controlled experiment.

\section{Future Directions}

The present study serves as preliminary research from which several different research questions could stem. For example, the implications of the current study could be made more pertinent to Americans today by experimenting with languages that are more commonly spoken in America, such as Spanish, French, or Chinese. Results from such a study could potentially assist an even wider range of ELLs in America, as it would be 
possible to identify the most common misperceptions native English speakers make while listening to words from those more common languages. Another possible future direction to take this study would be to assess native American English speakers' perceptions of non-native phonemes, rather than non-native phonotactic constraints. Phonemes, in addition to phonotactic constraints, vary widely across languages and are equally as critical to be able to accurately perceive as the phonotactic constraints when learning to speak and comprehend a new language. It would be interesting to determine if phonological knowledge has any influence on perceptions of non-native phonemes, despite having no influence on perceptions of non-native phonotactic constraints, according to the results of this study. This is a question worthy of investigation because it is reasonable to suppose that the knowledge possessed by individuals who have studied phonology regarding the articulatory features of English phonemes (i.e., manner of articulation, place of articulation, and voicing) would enhance their abilities to distinguish such features of phonemes from other languages.

\section{Conclusion}

Due to ever-increasing linguistic diversity, it is necessary to understand differences among the phonemes that comprise different languages and the rules for how those phonemes are used within a given language to form words. This study found that phonological knowledge did not influence a native American English-speaker's person's perception of non-native phonotactic constraints. An analysis of misperception of phonemes revealed that the most common misperception made was the omission of one phoneme when two were illegally combined. The results of this study, though not consistent with anticipated results, have many implications for issues concerning the linguistic diversity of the United States, among other issues related to language.

\section{References}

Callan, D. E., Jones, J. A., Callan, A. M., \& Akahane-Yamada, R. (2004). Phonetic perceptual identification by native- and second- language speakers differentially activates brain regions involved with acoustic phonetic processing and those involved with articulatory-auditory/orosensory internal models. NeuroImage, 22, 1182-1194.

Lentz, T. O., \& Kager, R. W. J. (2015). Categorical phonotactic knowledge filters second language input, but probabilistic phonotactic knowledge can still be acquired. Language and Speech, 58(3), 387-413.

Messier, J., \& Jackson, C. W. (2014). A comparison of phonemic and phonological awareness in educators working with children who are d/Deaf or hard of hearing. American Annals of the Deaf, 158(5), 522-538.

Onishi, K. H., Chambers, K. E., \& Fisher, C. (2002). Learning phonotactic constraints from brief auditory experience. Cognition, 83, B13-B23.

Polka, L. (1992). Characterizing the influence of native language experience on adult speech perception. Perception \& Psychophysics, 52(1), 37-52.

Spencer, E. J., Schuele, C. M., \& Guillot, K. M. (2008). Phonemic awareness skill of speech-language pathologists and other educators. Language, Speech, and Hearing Services in Schools, 39, 512-520. 